Preprint of the paper:

"Meshless methods applied to potential flow problems"

G. Mosqueira, I. Colominas, F. Navarrina, M. Casteleiro (2001)

En "Computational Fluid and Soild Mechanics", 908---911; K.J.Bathe (Ed.); Elsevier, Oxford, UK. (ISBN: 0-08-043944-6)

http:/caminos.udc.es/gmni 


\title{
Meshless methods applied to potential flow problems
}

\author{
Gonzalo Mosqueira*, Ignasi Colominas, Fermín Navarrina, Manuel Casteleiro \\ Department of Applied Mathematics, Civil Engineering School, Universidade da Coruña, Campus de Elviña, 15192 La Coruña, Spain
}

\begin{abstract}
In this paper we discuss some of the meshless approaches that have been recently proposed for solving boundary value problems. Several functional approximations and different integration schemes are studied. In this research, we apply this kind of techniques to potential flow problems and we analyze the advantages and disadvantages of the use of these methods.
\end{abstract}

Keywords: Meshless methods; Weighted least squares interpolation; Smooth particle hydrodynamics; Partition of unity; Enrichment functions; Potential flow

\section{Introduction}

Under the name 'meshless methods' a set of numerical techniques to solve differential equations are included. Its main purpose is to avoid the rigid connectivity typical of other methods like the finite element or the finite differences methods [1].

The most important advantages of these techniques appear, essentially, in two kinds of problems: those with very complicated domains, where the mesh generation process may become extremely complicated; and in a variety of problems related with large deformations, moving boundaries or discontinuities. The high refinement needed in these cases increases the mesh generation cost and implies a computational effort that can be unaffordable.

In the same way as in the finite element method, the first step to construct a meshless method is the generation of a local approximation to the solution in terms of the nodal values. In general, this local character can be obtained by means of the so-called kernel approximation or by the use of a weighted least squares interpolation. The first technique is used, for example, in the Smooth Particle Hydrodynamics method (SPH) [2] or in the Reproducing Kernel Particle Method (RKPM) [3]. The Diffuse Element Method and the Element Free Galerkin Method include the second one [1].

Another important item related with the construction of the meshless methods is the integration scheme used to solve a boundary value problem. Several alternatives have been studied to deal with this question. Some of them need

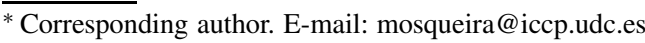

the existence of an underlying mesh [1]. In other cases, this mesh is avoided by using a point collocation approach [4] or a nodal integration scheme [2].

The practical cases solved with this kind of methods have shown that most of them imply a high computational cost, what reduces their range of applicability. For this reason, some new ideas have been recently proposed, such as combining finite elements and meshless interpolations (enrichment of finite elements with the element free Galerkin method [5], or with the reproducing kernel particle method [3]) and developing meshless methods on the basis of the partition of unity concept ( $h p$-clouds method, partition of unity finite element method), with the aim of providing an efficient way to perform $h-p$ adaptivity.

One of the advantages of the partition of unity concept is that it allows to include a priori knowledge about the solution. In this way, we have investigated the use of enrichment procedures applied to weighted least squares approximations with point collocation approaches. The good behavior of this technique has been checked on potential problems with the presence of high gradients [4]. As a continuity of this research, a meshless formulation applied to potential flow problems will be presented in this paper.

\section{Basis of meshless approximations}

A meshless approximation is characterized by the absence of predefined connectivities as in finite elements or finite differences. This property can be initially stated both from a continuous and a discrete point of view. 
Given a domain $\Omega$, an approximation $u^{h}(\boldsymbol{x})$ to a function $u(\boldsymbol{x})$ can be written in terms of the values of this function in a certain area, defined by $\boldsymbol{r} \in \Omega$ and weighted with a function $K(\boldsymbol{x}, \boldsymbol{r})$, in the following manner:

$$
\begin{aligned}
u(\boldsymbol{x}) \approx u^{h}(\boldsymbol{x}) & =\int_{r \in \Omega} K(\boldsymbol{x}, \boldsymbol{r}) u(\boldsymbol{r}) \mathrm{d} \Omega \\
& \approx \sum_{i_{p}=1}^{n_{p}} K\left(\boldsymbol{x}, \boldsymbol{r}_{i_{p}}\right) u\left(\boldsymbol{r}_{i_{p}}\right) V_{i_{p}}(\boldsymbol{x}),
\end{aligned}
$$

being $V_{i_{p}}(\boldsymbol{x})$ the integration weights at each integration point $i_{p}$.

If each integration point is understood as one of the $n_{p}$ nodal points in which the domain is discretized, Eq. (1) can be written using a FEM type approximation:

$\sum_{i_{p}=1}^{n_{p}} K\left(\boldsymbol{x}, \boldsymbol{r}_{i_{p}}\right) u\left(\boldsymbol{r}_{i_{p}}\right) V_{i_{p}}(\boldsymbol{x})=\sum_{i_{p}=1}^{n_{p}} N_{i_{p}}(\boldsymbol{x}) u_{i_{p}}$,

being $u_{i_{p}}=u\left(\boldsymbol{r}_{i_{p}}\right)$ and $N_{i_{p}}(\boldsymbol{x})=K\left(\boldsymbol{x}, \boldsymbol{r}_{i_{p}}\right) V_{i_{p}}(\boldsymbol{x})$.

Depending on the applications, the trial functions $N_{i_{p}}(\boldsymbol{x})$ are required to verify some properties. In the problems we are treating, the most important ones are: that the local character of the approximation must be guaranteed and that the partition of unity must be fulfilled. There are many ways to achieve these goals and every case gives rise to a different meshless method.

In general, the local character can be obtained by the choice of an adequate weighting function $K\left(\boldsymbol{x}, \boldsymbol{r}_{i_{p}}\right)$. A good one is that which takes its maximum value at the given point $\boldsymbol{x}$ and vanishes outside a selected surrounding region. For example:

$K(\boldsymbol{x}, \boldsymbol{r})= \begin{cases}\left.W(z)\right|_{z=|(\boldsymbol{r}-\boldsymbol{x}) / \rho|}>0, & \text { if } \boldsymbol{r} \in B(\boldsymbol{x}) ; \\ 0, & \text { otherwise, }\end{cases}$

$W(z)$ being a gaussian, a spline or any other truncated function, and $B(\boldsymbol{x})$ a selected suitable subdomain in the neighborhood of the given point $\boldsymbol{x}$. Hence, in this case, the dilation parameter $\rho$ plays an important role in Eq. (3), since it contributes to characterizing the support of the weighting function [3].

In the same way, the consistency properties depend on the trial functions $N_{i_{p}}(\boldsymbol{x})$. So, once the function $K\left(\boldsymbol{x}, \boldsymbol{r}_{i_{p}}\right)$ is fixed, the choice of the integration weights $V_{i_{p}}(\boldsymbol{x})$ is essential. One alternative is to impose that $u^{h}(\boldsymbol{x})$ approaches $u(\boldsymbol{x})$ in a weighted least squares sense [4]. In this case, $V_{i_{p}}(\boldsymbol{x})$ can be defined as:

$V_{i_{p}}(\boldsymbol{x})=\boldsymbol{p}^{t}(\boldsymbol{x}, \boldsymbol{x})\left\langle\boldsymbol{p}, \boldsymbol{p}^{t}\right\rangle^{-1} \boldsymbol{p}\left(\boldsymbol{x}, \boldsymbol{r}_{i_{p}}\right)$,

being $p(x, r)=\left.\varphi(z)\right|_{z=(r-x) / \rho}$, where $\varphi(z)$ is a complete base of selected interpolating functions (generally polynomials of a certain order) in $\boldsymbol{z}$ and $\left\langle\boldsymbol{p}, \boldsymbol{p}^{t}\right\rangle$ a scalar product weighted with the function $K(\boldsymbol{x}, \boldsymbol{r})$.

The integration weights defined in this way are used, for example, in the element free Galerkin method [1] or in the finite point method [6], and they guarantee that the partition of unity is fulfilled by the trial functions.

\section{Examples}

As an example, we apply the above meshless methods to a simple potential flow governed by Poisson's equation, with Neumann and Dirichlet boundary conditions:

$\begin{array}{ll}\Delta \phi+f=0 & \text { in } \Omega \\ \phi=\phi_{u} & \text { in } \Gamma_{u} \\ \nabla \phi \cdot \boldsymbol{n}=t & \text { in } \Gamma_{t}\end{array}$

The application of the weighted-residuals method leads to the variational form of the above stated problem in terms of the trial approximation $u^{h}$ to the solution $u$

$$
\begin{gathered}
\int_{\boldsymbol{r} \in \Omega} \omega_{j_{p}}[\phi+f] \mathrm{d} \Omega+\int_{\boldsymbol{r} \in \Gamma_{u}} \omega_{j_{p}}^{u}\left[\phi-\phi_{u}\right] \mathrm{d} \Gamma \\
+\int_{\boldsymbol{r} \in \Gamma_{t}} \omega_{j_{p}}^{t}[\nabla \phi \cdot \boldsymbol{n}-t] \mathrm{d} \Gamma=0
\end{gathered}
$$

which must hold for the test functions $\left\{\omega_{j_{p}}\right\},\left\{\omega_{j_{p}}^{t}\right\}$ and $\left\{\omega_{j_{p}}^{u}\right\}$ defined on $\Omega, \Gamma_{t}$ and $\Gamma_{u}$, respectively, where $j_{p}=$ $1, \ldots, n_{p}$.

For a given set of $n_{p}$ trial functions defined on $\Omega$ the approximation $u^{h}$ to the solution $u$ can be discretized as $u(\boldsymbol{x}) \approx u^{h}(\boldsymbol{x})=\sum_{i_{p}=1}^{n_{p}} N_{i_{p}}(\boldsymbol{x}) u_{i_{p}}$, where $n_{p}$ are the total scattered points of the solution domain, and the trial functions $N_{i_{p}}(\boldsymbol{x})$ can be constructed by using the above methodology.

Now, we can derive different numerical formulations from the variational form of Eq. (6). In order to take advantage of the meshless character of the approximation we can use a point-collocation approach [4] $\left(\omega_{j}=\omega_{j}^{t}=\right.$ $\omega_{j}^{u}=\delta\left(\boldsymbol{r}-\boldsymbol{r}_{j_{p}}\right)$, where $\delta(\cdot)$ is the Dirac delta) or some kind of nodal integration [2]. Several different approaches have been proposed by other authors, but some kind of auxiliary grid is then required to evaluate the resulting integrals. In any case, regardless of the integration scheme taken, a system of linear equations is obtained.

In the following example we show some results obtained by using these formulations. The potential problem we solve is:

$$
\begin{array}{ll}
\Delta V=0, & L_{0} \leq \sqrt{x^{2}+y^{2}} \leq L_{1}, \\
\left.V(x, y)\right|_{\sqrt{x^{2}+y^{2}}=L_{0}}=1, & \left.V(x, y)\right|_{\sqrt{x^{2}+y^{2}}=L_{1}}=\sin (\theta),
\end{array}
$$

The analytical solution depends on $\ln \sqrt{x^{2}+y^{2}}$. If we use this function as an enrichment function, for a given set of $n_{p}$ trial functions $N_{i_{p}}$ defined on the domain, the approximations $V^{h}$ to the solution $V$ can be written as: 


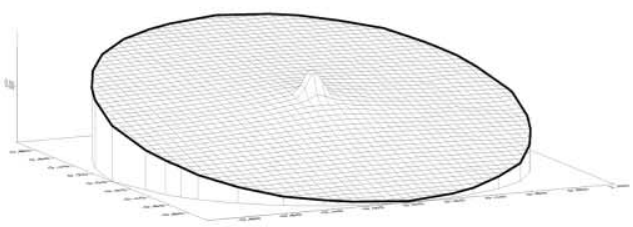

Figure a
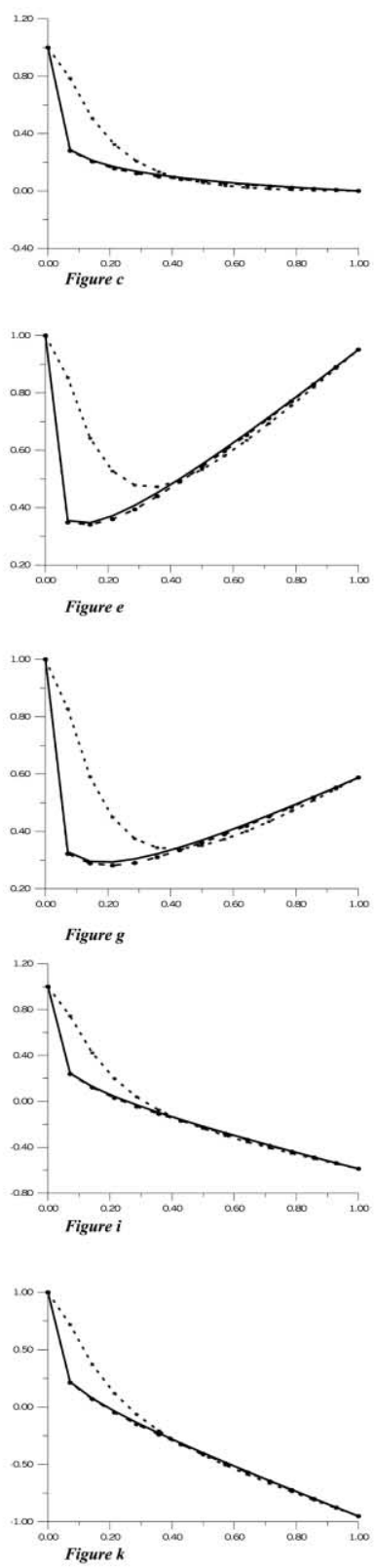

Angle: $0 \mathrm{rad}$. Points of subdomain: 16
Nodal points: 225

$\because$ WLS without enrichment

:Collocation points: 225

WLS with total

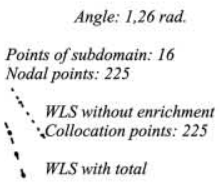

WLS with total

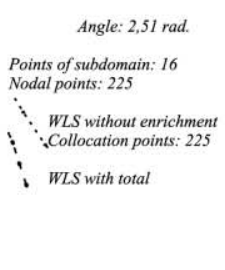

Angle: $3,77 \mathrm{rad}$

Points of subdomain: 16 Nodal points: 225

$\because$ WLS without enrichment

:Collocation points: 225

WLS with total

Points of subdomain: 16 Nodal points: 225

$\because$ WLS without enrichment

$\because$ Collocation points: 225

WLS with total
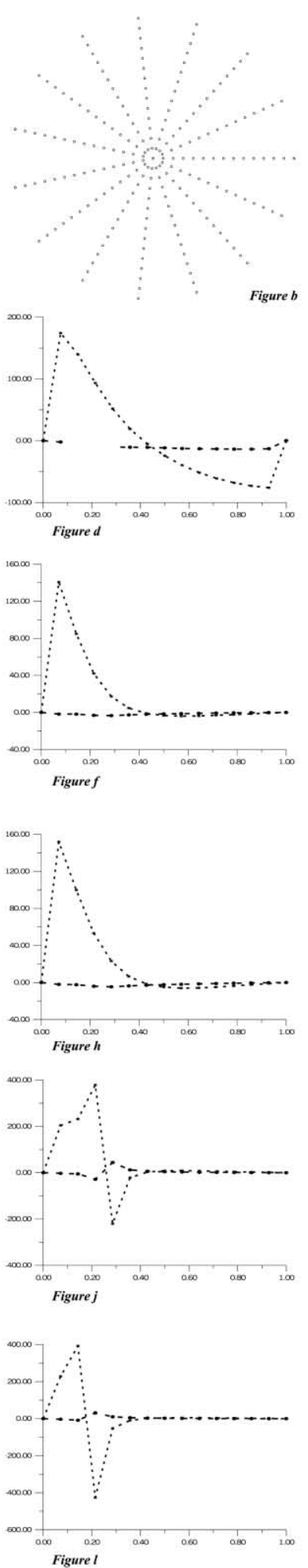

Fig. 1. 2D numerical example: comparison of results obtained by using different WLS formulations. 
Without enrichment functions:

$V^{h}=\sum_{i_{p}=1}^{n_{p}} N_{i_{p}} u_{i_{p}}$.

With enrichment functions:

$V^{h}=\sum_{i_{p}=1}^{n_{p}} N_{i_{p}}\left(u_{i_{p}}+k_{i_{p} 1} F_{1}(r)\right)$.

Total enrichment:

$F_{1}(r)=\ln \left(\sqrt{x^{2}+y^{2}}\right), \quad L_{0} \leq \sqrt{x^{2}+y^{2}} \leq L_{1}$.

Local enrichment:

$\begin{array}{ll}F_{1}(r)=0, & L_{0} \leq \sqrt{x^{2}+y^{2}}<r_{0} ; \\ F_{1}(r)=\ln \left(\sqrt{x^{2}+y^{2}}\right), & r_{0} \leq \sqrt{x^{2}+y^{2}} \leq r_{1} ; \\ F_{1}(r)=0, & r_{1}<\sqrt{x^{2}+y^{2}} \leq L_{1} .\end{array}$

In the above expression, $n_{p}$ is the total number of nodal points of the solution domain. The weighting function that has been used is the truncated gaussian.

In Fig. 1, we present the analytical solution $(a)$ and the results obtained when 225 nodal points (see $b$ ) are used. In this case, we compare the analytical solution and the approximations obtained by using the weighted least squares approach with or without enrichment functions along five different radial lines $(c, e, g, i, k)$. The relative errors are compared in figures $d, f, h, j, l$.

At this moment, we are studying the behavior of other integration schemes applied to this kind of problems. Our goal is to avoid the disadvantages of the point-collocation approaches but preserving the meshless character of the method.

\section{Acknowledgements}

This project is partially supported by the 'Ministerio de Ciencia y Tecnología (\#1FD97-0108)' of the Spanish Government, co-financed with FEDER funds, by the power company 'Unión Fenosa Ingeniería S.A.', and by research fellowships of the 'Xunta de Galicia' and the 'Universidad de La Coruña'.

\section{References}

[1] Belystchko T, Krongauz Y, Organ D, Krysl P. Meshless methods: An overview and recent developments. Comput Methods Appl Mech Eng 1996;139:3-48.

[2] Bonet J, Lok TSL. Variational and momentum preserving aspects of Smooth Particle Hydrodynamics formulations. Comput Methods Appl Mech Eng 1998;submitted.

[3] Liu WK, Li S, Belytschko T. Moving least square reproducing kernel methods, I. Methodology and convergence. Comput Methods Appl Mech Eng. 1997;143:113-154.

[4] Mosqueira G, Colominas I, Navarrina F, Casteleiro M. An enriched meshless numerical approach for potential theory problems. Proc Eur Congr Computational Methods in Applied Sciences and Engineering, ECCOMAS 2000, Barcelona, 2000.

[5] Huerta A, Fernández-Méndez S. Enrichment of the finite element method with meshless methods. VI Congr Nacl Mecânica Aplicada e Computacional, 2000, Vol 1, pp. 6170.

[6] Taylor RL, Idelsohn S, Zienkiewicz OC, Oñate E. Moving Least Square Approximation for Solution of Differential Equations. Research Report 74, CIMNE, Barcelona, 1995. 